\title{
ND-Wavelets Derived from Anti-symmetric Systems of Isolated Particles using the Determinant of Slater
}

\author{
H. M. de Oliveira and V. V. Vermehren
}

\begin{abstract}
Wavelets are known to be closely related to atomic orbital. A new approach of 2D, 3D and multidimensional wavelet system is proposed from a paralell with anti-symmetric systems of several isolated particles. The theory of fermionic states is used to generate new $n$-dimensions wavelets, $n \geq 2$, by the determinant of Slater. As pioneering paper in exchanging formalism between particle wave-functions and wavelets, it opens some perspectives for further adaptations derived from the physics of particles in the wavelet analysis scope.
\end{abstract}

Keywords-3D wavelets, anti-symmetric wavelets, orbital wavelets, image analysis.

\section{INTRODUCTION}

Wavelet transforms are important tools in image processing due to their capabilities of multiresolution analysis and image decomposition [6], [18]. Wavelet-based image processing is a routinely and largely adopted approach in texture image decomposition, subband coding, fast image segmentation [1], [3], [13], [16], [31], even for 3D [30]. There are also plenty of applications of wavelet in computer graphics [20], [23], [25], [27].

There is a rooted [2], but not fully explored link between quantum mechanics and wavelets. The wave nature of light can be deduced from the phenomenon of interference, the photoelectric effect, however, seems to suggest a corpuscular nature of light. Theoretical physicists struggled to include observations like the photoelectric effect and the wave-particle duality into their formulations [29]. Erwin Schrödinger, an Austrian physicist, was using advanced mechanics to deal with these phenomena and developed a equation that relates the space-time in quantum mechanics, in an attempt to get the analog of the classical mechanics. Because wavelets are localized in both time and frequency they offer significant advantages for the analysis of problems in quantum mechanics. In this paper, we shed some light on some of these relations. Rather than seeking at wavelet features on particles or waves, we adapted some concepts of quantum mechanics to a novel wavelet decomposition for still images.

Initially, we introduce in Section [1] the motivation to build these wavelets, with inspiration in "Particle Physics" [4], [11]. In Section III, we introduce the construction of orbital wavelets for the image decomposition, starting from a single mother wavelet. It is shown this construction actually generates twodimensional wavelets. Section IV includes the main lines for an extension to the 3D-case and to higher dimensions. Finally, the concluding remarks are presented in Section VI.

Statistics Department, Federal University of Pernambuco (UFPE), Recife-PE, Brazil, E-mail: hmo@de.ufpe.br. Electrical Engineering Department, University of the State of Amazonas, Manaus, Brazil, victor.vermehren@gmail.com

\section{Motivation Arising From Particle Physics}

Usual wavelet image analysis combines one-dimensional (1D) wavelets to generate a two-dimensional (2D) wavelet [1], [31]. This is equivalent to the analysis by the matrix:

$$
\left[\begin{array}{ll}
L L & H L \\
L H & H H
\end{array}\right]
$$

where $L$ and $H$ denote low- and high-pass bands, respectively [28].

The combinations $L L$ and $H H$ naturally exhibit a symmetry (even): $\varphi(x) \cdot \varphi(y)=\varphi(y) \cdot \varphi(x)$, ditto for $\psi(x) \cdot \psi(y)=\psi(y)$. $\psi(x)$. Now, the combination of $\varphi(\cdot)$ and $\psi(\cdot)$ resulting in two different analyzes and non-commutative, namely, $\varphi(x) \cdot \psi(y)$ and $\psi(x) \cdot \varphi(y)$. The combination of $\varphi(\cdot)$ and $\psi(\cdot)$ should result in asymmetry (odd symmetry), and the exchange of the coordinates $x \leftrightarrow y$ just swap the direction of observation. The combined $\varphi_{-} \psi$ wave should be such that:

$$
\varphi_{-} \psi(x, y)=-\varphi_{-} \psi(y, x)
$$

The proposal then is to define the orbital combination between $\varphi$ and $\psi$ by the following wave function:

$$
\varphi_{-} \psi(x, y):=\frac{1}{\sqrt{2}}[\varphi(x) \psi(y)-\psi(x) \varphi(y)],
$$

instead of merely $\varphi(x) \cdot \psi(y)(L H)$ or $\psi(x) \cdot \varphi(y)(H L)$. It should be noted that it now hold Equation (2).

Worth nothing that the subtraction of the standard images $L H$ - $H L$ results in exactly the picture of the orbital decomposing, as well as its negative, on the secondary diagonal of the decomposition matrix of Equation (1).

\section{ORbital WAVElet For the 2D CASE}

The wave functions describing electronic orbital can be combined generating "atomic orbitals". The "equivalent" in the scope of wavelets, also characterized by wave functions, would be combination of different spatial wavelets [7]. In the case of particles is typically assumed the combination of antisymmetric particles without interaction [11]. The combination of $\alpha$ and $\beta$ states should not depend on which of the particles ( 1 or 2$)$ is in one of the particular states. This is called "exchange degeneracy". It corresponds to a probability density of two particles, being one in the alpha state and another in the beta state not knowing where a particular state. There are two ways to achieve this [10]:

$$
\psi_{S}\left(r_{1}, r_{2}\right):=\frac{1}{\sqrt{2}}\left[\psi_{\alpha \beta}\left(r_{1}\right)+\psi_{\beta \alpha}\left(r_{2}\right)\right]
$$


and

$$
\psi_{A}\left(r_{1}, r_{2}\right):=\frac{1}{\sqrt{2}}\left[\psi_{\alpha \beta}\left(r_{1}\right)-\psi_{\beta \alpha}\left(r_{2}\right)\right] .
$$

where $r_{1}$ and $r_{2}$ are the positions of particle 1 and 2 , and $\alpha$ and $\beta$ are particular states, respectively.

This idea can be used in the decomposition of Equation (1). $\psi_{S}$ is employed in the main diagonal and $\psi_{A}$ in the secondary diagonal. Interestingly, employing the combination symmetric diagonally main results in

$$
L L(x, y):=\frac{1}{\sqrt{2}}\left[\varphi^{*}(x) \varphi(y)+\varphi^{*}(y) \varphi(x)\right]
$$

and

$$
H H(x, y):=\frac{1}{\sqrt{2}}\left[\psi^{*}(x) \psi(y)+\psi^{*}(y) \psi(x)\right] .
$$

This definition allows, in particular, analyzing images using continuous complex wavelets (see also [14]). In the case of real wavelets, simplification collapses to the usual $\varphi(x) \cdot \varphi(y)$ or $\psi(x) \cdot \psi(y)$. For the sake of simplicity, we often drop the variables $x$ and $y$ and denote by $(L L)$ and $(H H)$ as introduced in Equation (1). The orbital-based 2D analysis is shown in Appendix.

The initial proposal for "combination" of two wavelets was adopted for image analysis (2D) considering the same orthogonal mother wavelet, but at different scales of multiresolution. The two wavelets are $\psi_{a_{1}, b}($.$) and \psi_{a_{2}, b}($.$) and$ the approach for simultaneous analysis in two different scales corresponds to the following set-up: Consider a 1D orthogonal [7], [15], [17] wavelet mother $\psi(x)$ equipped with her daughters $\left\{\psi_{a, b}(x)\right\}_{a \neq 0, b \in R}$.

Definition 1: The function 2D-orbital at the scales $\left\{a_{1}, a_{2}\right\}$ is defined by:

$$
\psi_{A}(x, y):=\frac{1}{\sqrt{2}} \operatorname{det}\left[\begin{array}{ll}
\psi_{a_{1}, b}^{*}(x) & \psi_{a_{1}, b}^{*}(y) \\
\psi_{a_{2}, b}(x) & \psi_{a_{2}, b}(y)
\end{array}\right]
$$

what can be rewritten as

$$
\begin{aligned}
\psi_{A}(x, y)= & \frac{1}{\sqrt{2\left|a_{1}\right|\left|a_{2}\right|}} \psi^{*}\left(\frac{x-b}{a_{1}}\right) \psi\left(\frac{y-b}{a_{2}}\right) \\
& -\frac{1}{\sqrt{2\left|a_{1}\right|\left|a_{2}\right|}} \psi\left(\frac{x-b}{a_{2}}\right) \psi^{*}\left(\frac{y-b}{a_{1}}\right) .
\end{aligned}
$$

The condition $a_{1} \neq a_{2}$ eliminates the degeneration into $\psi_{A}(x, y)=0$. This is to some extent in connection to the Pauli Exclusion Principle [10]. It state that with a singlevalued many-particle wavefunction is equivalent to requiring the wavefunction to be antisymmetric. An antisymmetric twoparticle state is represented as a sum of states in which one particle is in state $\alpha$ and the other in state $\beta$. Besides, the relationship $\psi_{A}(y, x)=-\psi_{A}(x, y)$ ensures the desired asymmetry. Here, we use the same wavelet-mother, but on different scales. It will be seen that the orthogonality requirements correspond to the "dual" of particles without interaction. If the wavelets are orthogonal on any two scales, one can perform a decomposition of an image "simultaneously" in both scales. The decomposition 2D sated in Definition 1 results in a strict 2D-wavelet.

Hypothesis 1: If the wavelets $\left\{\psi_{a, b}(t)\right\}$ are orthogonal, then the inner product $\left\langle\psi_{a_{1}, b}, \psi_{a_{2}, b}\right\rangle=0$ and the following integrals cancel out $\forall a_{1} \neq a_{2}$ :

$$
\int_{-\infty}^{\infty} \psi_{a_{1}, b}(x) \cdot \psi_{a_{2}, b}^{*}(x) \mathrm{d} x=\int_{-\infty}^{\infty} \psi_{a_{1}, b}^{*}(x) \cdot \psi_{a_{2}, b}(x) \mathrm{d} x=0 .
$$

It is also noteworthy that

$$
\left\langle\psi_{a_{1}, b}, \psi_{a_{2}, b}\right\rangle^{*}=\left\langle\psi_{a_{2}, b}, \psi_{a_{1}, b}\right\rangle
$$

Proposition 1: The previously defined 2D-orbital function has oscillatory behavior satisfying the following properties:

1) $\int_{-\infty}^{\infty} \psi_{A}(x, y) \mathrm{d} x=0$

2) $\int_{-\infty}^{\infty} \psi_{A}(x, y) \mathrm{d} y=0$

3) $\int_{-\infty}^{\infty} \int_{-\infty}^{\infty} \psi_{A}(x, y) \mathrm{d} x \mathrm{~d} y=0$.

Proof. It follows that

$\int_{-\infty}^{\infty} \psi_{A}(x, y) \mathrm{d} x=\frac{1}{\sqrt{2}} \psi_{a_{2}, b}(y) \cdot \overline{\psi_{a_{1}, b}^{*}(x)}-\frac{1}{\sqrt{2}} \overline{\psi_{a_{2}, b}(x)} \cdot \psi_{a_{1}, b}^{*}(y)$,

where

$$
\overline{\psi_{a, b}(x)}=\int_{-\infty}^{\infty} \psi_{a, b}(x) \mathrm{d} x .
$$

Therefore item 1 derives from the fact that $\psi_{a, b}(x), a=$ $\left\{a_{1}, a_{2}\right\}$ be individual wavelets. Demonstration of item 2 is similar, considering that

$$
\begin{array}{r}
\int_{-\infty}^{\infty} \psi_{A}(x, y) \mathrm{d} y= \\
\frac{1}{\sqrt{2}} \overline{\psi_{a_{2}, b}(y)} \cdot \psi_{a_{1}, b}^{*}(x)-\frac{1}{\sqrt{2}} \psi_{a_{2}, b}(x) \cdot \overline{\psi_{a_{1}, b}^{*}(y)} .
\end{array}
$$

Now the condition $\int_{-\infty}^{\infty} \int_{-\infty}^{\infty} \psi_{A}(x, y) \mathrm{d} x \mathrm{~d} y=0$ follows from Fubini's theorem [24], regardless of the order of integration.

Proposition 2: The 2D-orbital functions have normalized energy.

Proof. Computing $\left|\psi_{A}(x, y)\right|^{2}=\psi_{A}(x, y) \cdot \psi_{A}^{*}(x, y)$, we arrive at:

$\left|\psi_{A}(x, y)\right|^{2}=\frac{1}{2}\left|\psi_{a_{1}, b}(x)\right|^{2} \cdot\left|\psi_{a_{2}, b}(y)\right|^{2} \times\left|\psi_{a_{1}, b}(y)\right|^{2} \cdot\left|\psi_{a_{2}, b}(x)\right|^{2}$ 


$$
\int_{-\infty}^{\infty} \int_{-\infty}^{\infty}\left|\psi_{A}(x, y)\right|^{2} \mathrm{~d} x \mathrm{~d} y=1
$$

If, by hypothesis $\psi_{a_{1}, b}(t) \perp \psi_{a_{2}, b}(t)$, then all cross terms are void, concluding the proof. It is possible (more easily) to combine orthogonal 1D-wavelets and use them to build a new 2D-wavelet.

Proposition 3: The 2D-orbital function is a 2D wavelet.

Proof. Starting from $\psi(t) \leftrightarrow \Psi(w)$ and the fact that the admissibility condition holds [5], [6],

$C_{\psi}:=\int_{-\infty}^{\infty} \frac{|\Psi(w)|^{2}}{|w|} d w<+\infty$, and similarly for their daughter wavelets $\psi_{a, b}(t) \leftrightarrow \Psi_{a, b}(w)$ results in $\int_{-\infty}^{\infty} \frac{\left|\Psi_{a, b}(w)\right|^{2}}{|w|} d w<\infty$, since $\Psi_{a, b}(w)=\sqrt{|a|} \Psi(a w) e^{-j w b}$ $[8]$. Let us now evaluate the condition for the $2 \mathrm{D}$ case. If the Fourier transform pair $\psi_{A}(x, y) \leftrightarrow \Psi_{A}(u, v)$ do exist, the 2Dspectrum of $\psi_{A}$ can be computed in terms of the 1D-spectrum of $\psi$ :

$$
\Psi_{A}(u, v)=\frac{\sqrt{\left|a_{1} a_{2}\right|}}{\sqrt{2}}\left[\Psi\left(a_{1} u\right) \Psi^{*}\left(a_{2} v\right)-\Psi\left(a_{2} u\right) \Psi^{*}\left(a_{1} v\right)\right]
$$

From the generalized Parseval-Plancherel energy theorem [6], [24], the cross-terms vanish due to the orthogonality, and

$$
\begin{array}{r}
\left|\Psi_{A}(u, v)\right|^{2}= \\
\frac{\left|a_{1} a_{2}\right|}{2}\left|\Psi\left(a_{1} u\right)\right|^{2}\left|\Psi\left(a_{2} v\right)\right|^{2}+\frac{\left|a_{1} a_{2}\right|}{2}\left|\Psi\left(a_{2} u\right)\right|^{2}\left|\Psi\left(a_{1} v\right)\right|^{2} .
\end{array}
$$

Letting

$$
C_{\psi_{A}}:=\iint_{-\infty}^{\infty} \frac{\left|\Psi_{A}(u, v)\right|^{2}}{|u| \cdot|v|} \mathrm{d} u \mathrm{~d} v
$$

the proof that $C_{\psi_{A}}<+\infty$ follows directly from the marginal admission conditions of the 1D daughter-wavelets.

\section{THE 3D-Orbital WAVELETS}

For extension of the results in the 3D case (or even higher dimensions), one might consider the following definition inspired by the "Slater determinant" [26], which is used for antisymmetric systems of several isolated particles (fermionic state).

Definition 2: The 3D-orbital function at three distinct scales $\left\{a_{1}, a_{2}, a_{3}\right\}$ is given by

$$
\psi_{A}(x, y, z)=\frac{1}{\sqrt{3 !}} \cdot \operatorname{det}\left[\begin{array}{ccc}
\psi_{a_{1}, b}^{*}(x) & \psi_{a_{1}, b}^{*}(y) & \psi_{a_{1}, b}^{*}(z) \\
\psi_{a_{2}, b}(x) & \psi_{a_{2}, b}(y) & \psi_{a_{2}, b}(z) \\
\psi_{a_{3}, b}^{*}(x) & \psi_{a_{3}, b}^{*}(y) & \psi_{a_{3}, b}^{*}(z)
\end{array}\right]
$$

The general case follows the same lines. Again, ensuring the orthogonality of the 1D wavelet chosen as the starting point is an essential statement. Further generalizations can also be done.
This paper presented a way to combine orthogonal wavelets, Equation (4) and (5), using a method similar to the LCAO (Linear Combination of Atomic Orbitals) approach [19]. When there is no orthogonality, one possible solution is to consider the quantity $S \neq 0$, called orbital overlap (or recoating), which defined by:

$$
S:=\int_{-\infty}^{\infty} \psi_{a_{1}, b}(x) \cdot \psi_{a_{2}, b}^{*}(x) \mathrm{d} x
$$

In this cases Equation (4) and Equation (5) are replaced by

$$
\widetilde{\psi_{S}}\left(r_{1}, r_{2}\right):=\frac{1}{\sqrt{2(1+S)}}\left[\psi_{\alpha \beta}\left(r_{1}\right)+\psi_{\beta \alpha}\left(r_{2}\right)\right],
$$

and

$$
\widetilde{\psi_{A}}\left(r_{1}, r_{2}\right):=\frac{1}{\sqrt{2(1-S)}}\left[\psi_{\alpha \beta}\left(r_{1}\right)-\psi_{\beta \alpha}\left(r_{2}\right)\right] .
$$

This can clearly be put as a generalization on Definition 1, but now in the wavelet framework. Since LCAO is larged and successful used in molecule studies, this similar wavelet approach can has a potential use.

\section{CONCluding Remarks}

Even prospective and introductory, the main ideas presented here can be explored, taking advantage of the cornucopia of tools used in particle physics and atomic orbitals theory. This paper offers an original and general approach for image decomposition engendered by asymmetric orthogonal wavelets, which allows much room, somewhat akin to the extension from wavelet to wavelet packets. Despite the focus being essentially on still image, this approach allows a fully scalable multimedia decomposition. It remains to be investigated the potential of this approach in image compressing [9], in 3D processing and scalable coding for multimedia schemes [21]. Applications in other scenarios such as wavelet-based watermarking [12] or steganography [22] also deserve an investigation. As pioneering paper in exchanging formalism between particle wave-functions and wavelets, it opens new perspectives for adaptations derived from the quantum chemistry in the wavelet analysis scope.

\section{ACKNOWLEDGEMENT}

The authors are grateful to Dr. Renato Cintra (UFPE Department of Statistics) who has actively contributed to the development of the main concepts related to the 2D-wavelet model linked to atomic orbitals. They also thanks to Dr. R. Ospina (UFPE Department of Statistics) for valuable support on LTTE $_{\mathrm{E}} \mathrm{X}$ issues. 


\section{REFERENCES}

[1] Antonini, M., Barlaud, M., Mathieu, P., and Daubechies, I. (1992). Image coding using wavelet transform. IEEE Transactions on Image Processing, $1: 205-220$.

[2] Ashmead, J. (2012). Morlet wavelets in quantum mechanics. Quanta, 1:58-70.

[3] Aujol, J. F., Gilboa, G., Chan, T., and Ocher, S. (2006). Structure-texture image decomposition: modeling, algorithms, and parameter selection. International Journal of Computer Vision, 67:111-136.

[4] Beiser, A. (1994). Concepts of Modern Physics. McGraw-Hill.

[5] Boggess, A. and Narcowich, F. J. (2009). A First Course in Wavelets with Fourier Analysis. Wiley.

[6] Burrus, C. S., Gopinath, R. A., and Guo, H. (1998). Introduction to Wavelets and the Wavelet Transform - A Primer. Prentice-Hall.

[7] de Oliveira, H. M. (2007b). Análise de Sinais para Engenheiros: Uma Abordagem via Wavelets. Editora Brasport.

[8] de Oliveira, H. M. (2007a). Análise de Fourier e Wavelets: sinais estacionários e não estacionários. Ed. Universítária UFPE.

[9] DeVore, R., Jawwerth, B., and Lucier, B. (1995). Image compression through wavelet transform coding. IEEE Trans. on Information Theory, 38:719-746

[10] Duck, I. and Sudarshan, E. C. G. (1998). Pauli and the Spin-Statistics Theorem. World Scientific.

[11] Eisberg, R. M. (2007). Fundamentals of Modern Physics. J. Wiley \& Sons.

[12] Hu, J., Huang, J., Huang, D., and Shi, Y. Q. (2002). Image fragile watermarking based on fusion of multi-resolution tamper detection. Electronics Letters, 38:1512-1513.

[13] Kim, B. G., Shim, J. I., and Park, D. J. (2003). Fast image segmentation based on multi-resolution analysis and wavelets. Pattern Recognition Letters, 24:2995-3006.

[14] Kingsbury, N. (1999). Image processing with complex wavelets. Phil. Transactions of Royal Society London, A Mathematical, Physical and Engineering Sciences, 357:2543-2560.

[15] Lawton, W. M. (1991). Necessary and sufficient conditions for constructing orthonormal wavelet bases. Journal of Mathematic Physics, 32:5761.

[16] Lim, J. S. (1990). Two-Dimensional Signal and Image Processing. Prentice Hall.

[17] Maaß, S. (1996). Families of orthogonal 2D wavelets. Society for Industrial and Applied Mathematics - Journal of Applied Analysis, 27:1454-1481.

[18] Mallat, S. G. (1999). A Wavelet Tour of Signal Processing. Academic Press, 2nd edition.

[19] Manhan, B. M. and Myers, R. J. (1987). University Chemistry. AddisonWesley Longman.

[20] Muraki, S. (1993). Volume data and wavelet transforms. IEEE Computer Graphics and Applications, 13:50-56.

[21] Ohm, J. R., der Schaar, M. V., and Woods, J. W. (2004). Interframe wavelet coding: motion picture representation for universal scalability. Image Communication Signal Processing, 19:877-908.

[22] Rekik, S., Guerchi, D., Hamam, H., and Selouani, S. A. (2012). Audio steganography coding using the discrete wavelet transforms. International Journal of Computer Science and Security, 6:79-93.
[23] Roerdink, J. B. T. M., and Westenberg M. A. (1999). Wavelet-based volume visualization. Nieuw Archief voor Wiskunde, 17:149-158.

[24] Rudin, W. (1976). Principles of Mathematical Analysis, volume 62. McGraw-Hill.

[25] Schröder, P. (1996). Wavelets in computer graphics. Proceedings of the IEEE, 84:615-625.

[26] Slater, J. C. (1929). The theory of complex spectra. Physical Reviews, $34: 1293-1322$.

[27] Stollnitz, E. J. and Derose, T. D. (1996). Wavelets for Computer Graphics. Morgan-Kaufmann Pub.

[28] Stollnitz, E. J., DeRose, T. D., and Salesin, D. H. (1995). Wavelets for computer graphics: A primer. IEEE Computer Graphics and Applications, $15: 76-84$.

[29] Sullivan, D. M. (2003). Quantum Mechanics for Electrical Engineers. IEEE Series on Microelectronics Systems. Wiley.

[30] Taubman, D. and Zakhor, A. (1994). Multirate 3-D subband coding of video. IEEE Trans. on Image Processing, 3:572-588.

[31] Vetterli, M. and Kovacevic, J. (1990). Wavelets and Subband Coding. Prentice-Hall.

\section{APPENDIX}

A general alternative formulation of standard image (2D) wavelet decomposition can be carried out by the following

$$
\left[\begin{array}{ll}
L L & H L \\
L H & H H
\end{array}\right]
$$

where $L$ and $H$ denote low- and high-pass bands, respectively.

$$
\begin{aligned}
& L L=\varphi_{-} \varphi_{S}(x, y):=\frac{1}{\sqrt{2}} \operatorname{det}\left[\begin{array}{cc}
\varphi^{*}(x) & \varphi^{*}(y) \\
-\varphi(x) & \varphi(y)
\end{array}\right] . \\
& H H=\psi_{-} \psi_{S}(x, y):=\frac{1}{\sqrt{2}} \operatorname{det}\left[\begin{array}{cc}
\psi^{*}(x) & \psi^{*}(y) \\
-\psi(x) & \psi(y)
\end{array}\right] . \\
& L H=\varphi_{-} \psi_{A}(x, y):=\frac{1}{\sqrt{2}} \operatorname{det}\left[\begin{array}{cc}
\varphi^{*}(x) & \varphi^{*}(y) \\
\psi(x) & \psi(y)
\end{array}\right] . \\
& H L=\psi_{-} \varphi_{A}(x, y):=\frac{1}{\sqrt{2}} \operatorname{det}\left[\begin{array}{cc}
\psi^{*}(x) & \psi^{*}(y) \\
\varphi(x) & \varphi(y)
\end{array}\right] .
\end{aligned}
$$

The symmetries involved are:

$\varphi_{-} \varphi_{S}(x, y)=\varphi_{-} \varphi_{S}(y, x)$ and $\psi_{-} \psi_{S}(x, y)=\psi_{-} \psi_{S}(y, x)$, whereas the antisymmetries are $\varphi_{-} \psi_{A}(x, y)=-\varphi_{-} \psi_{A}(y, x)$ and $\psi_{-} \varphi_{A}(x, y)=-\psi_{-} \varphi_{A}(y, x)$. 